\title{
四元 $\mathrm{BiMnVO}_{5}$ 的合成、电子结构与可见光催化性能研究
}

\author{
张 弦，张策，姜文君，冯德强，姚 伟 \\ (中国空间技术研究院 钱学森空间技术实验室, 北京 100094)
}

摘 要: 光催化去除水体中的有机污染物在污水净化领域具有广阔的应用前景。本研究分别采用水热法和固相法合 成了四元 $\mathrm{BiMnVO}_{5}$ 可见光催化剂, 对催化剂的形貌结构和光学性质进行了表征分析, 并计算了其电子结构。结果 表明, 水热法可以快速合成高纯度、高结晶度的 $\mathrm{BiMnVO}_{5}$ 。 $\mathrm{BiMnVO}_{5}$ 为直接带隙半导体, 禁带宽度为 $1.8 \mathrm{eV}$, 与 第一性原理计算结果一致。态密度分析结果表明, 其光吸收可归因于从 $\mathrm{Mn} 3 \mathrm{~d} / \mathrm{O} 2 \mathrm{p}$ 到 $\mathrm{V} 3 \mathrm{~d}$ 的电子跃迁。光催化实 验结果显示, 水热法合成的 $\mathrm{BiMnVO}_{5}$ 催化剂的催化活性最高, 可见光照射 $4 \mathrm{~h}$ 后，亚甲基蓝的降解率为 $98 \%$ 。着基 自由基和光生空穴是光催化过程中的主要活性物。经过 5 次循环利用后,该催化剂对亚甲基蓝的降解率仍可达 $85 \%$, 且形貌结构不变, 表现出良好的稳定性。

关 键 词: $\mathrm{BiMnVO}_{5}$ 半导体; 水热法; 电子结构; 光催化

中图分类号: TQ174 文献标志码: A

\section{Synthesis, Electronic Structure and Visible Light Photocatalytic Performance of Quaternary BiMnVO}

\author{
ZHANG Xian, ZHANG Ce, JIANG Wenjun, FENG Deqiang, YAO Wei \\ (Qian Xuesen Laboratory of Space Technology, China Academy of Space Technology, Beijing 100094, China)
}

\begin{abstract}
Removal of organic pollutants from water bodies by photocatalysis has broad application prospects in the field of sewage purification. In this study, the quaternary $\mathrm{BiMnVO}_{5}$ was synthesized by hydrothermal method and solid-state reaction, respectively. The morphology, structure and optical properties of the catalysts were characterized, and the electronic structure was calculated. Results showed that the hydrothermal method can rapidly synthesize pure $\mathrm{BiMnVO}_{5}$ with high crystallinity. $\mathrm{BiMnVO}_{5}$ is a semiconductor with a direct band gap of $1.8 \mathrm{eV}$, which is consistent with the results of first-principles calculations. Analysis of the density of states further reveals that the optical absorption band are attributed to the transitions from $\mathrm{Mn} 3 \mathrm{~d} / \mathrm{O} 2 \mathrm{p}$ to $\mathrm{V} 3 \mathrm{~d}$. The photocatalytic results show that the $\mathrm{BiMnVO}_{5}$ synthesized by hydrothermal method exhibits the highest catalytic activity. About $98 \%$ Methylene Blue (MB) is degraded when exposed to visible light for $4 \mathrm{~h}$. The hydroxyl radicals and photo-generated holes are proved to be the main active substances in the photocatalytic process. After 5 cycles, it still maintains capacity of degrading $85 \% \mathrm{MB}$ in $4 \mathrm{~h}$ under visible light irradiation, while its morphology and structure remain unchanged, which indicates $\mathrm{BiMnVO}_{5}$ a good photocatalyst with reliable stability.
\end{abstract}

Key words: $\mathrm{BiMnVO}_{5}$ semiconductor; hydrothermal method; electronic structure; photocatalysis

收稿日期：2021-04-21；收到修改稿日期：2021-07-02;网络出版日期：2021-07-12

基金项目: 国家自然科学基金(22001263); 中国航天科技集团钱学森青年创新基金(Y-KC-JT-QXS-012)

National Natural Science Foundation of China (22001263); Qian Xuesen Youth Innovation Fund (Y-KC-JT-QXS-012)

作者简介: 张 弦(1989-), 男, 博士，高级工程师. E-mail: zhangxian@qxslab.cn

ZHANG Xian(1989-), male, PhD, senior engineer. E-mail: zhangxian@qxslab.cn

通信作者: 姚 伟, 研究员. E-mail: yaowei@qxslab.cn

YAO Wei, professor. E-mail: yaowei@qxslab.cn 
光催化降解有机污染物在污水净化领域具有广 阔的应用前景 ${ }^{[1]}$ 。钒酸铋 $\left(\mathrm{BiVO}_{4}\right)$ 是一种典型的半导 体光催化剂, 其禁带宽度为 $2.45 \mathrm{eV}$, 且具有化学性能 稳定和无毒等特点, 受到了广泛关注 ${ }^{[2-4]}$ 。在 $\mathrm{BiVO}_{4}$ 的众多物相中, 具有单斜白铇矿结构的 $\mathrm{BiVO}_{4}$ 催化 性能最优, 这是由于单斜相中 $\mathrm{Bi}-\mathrm{O}$ 多面体畸变程 度高, 有利于光生电子-空穴对的产生与分离 ${ }^{[5]}$ 。然 而, $\mathrm{BiVO}_{4}$ 仅吸收部分可见光, 太阳能利用率低。因 此, 如何提升这类材料的光吸收效率是当前研究的 重点。

通过掺杂可以调控 $\mathrm{BiVO}_{4}$ 的禁带宽度，提升光 吸收效率。Zhou 等 ${ }^{[6]}$ 通过 Co 掺杂成功将其禁带宽 度降低至 $2.39 \mathrm{eV}$, 光催化反应 $5 \mathrm{~h}$ 后, $\mathrm{MB}$ 降解率提 升 $20 \%$ 。 $\mathrm{He}$ 等 ${ }^{[7]}$ 采用模板法制备了 $\mathrm{Cu}$ 掺杂 $\mathrm{BiVO}_{4}$ 纳米棒, 获得的最低禁带宽度为 $2.02 \mathrm{eV}$ 。Regmi 等 ${ }^{[8-9]}$ 采用水热法合成了 $\mathrm{Fe}$ 或 $\mathrm{Ni}$ 掺杂的 $\mathrm{BiVO}_{4}$, 获 得的最小禁带宽度约为 $2.37 \mathrm{eV}$ 。此外, 研究人员还 尝试利用 $M o 、 W 、 I n 、 L n(L n=Y 、 L a 、 S m 、 G d)$ 等元 素对 $\mathrm{BiVO}_{4}$ 进行掺杂, 但未能有效减小其禁带宽 度 ${ }^{[10-17]}$ 。然而, 掺杂对禁带宽度的调控范围有限, 且 杂质能级通常是光生电子-空穴对的复合中心。因此, 探索基于 $\mathrm{BiVO}_{4}$ 的新型窄带隙半导体具有重要的科 学意义和实用价值。

向 $\mathrm{BiVO}_{4}$ 体系中引入合适的结构基元, 形成具 有不同结构功能区的 Bi-M-V-O 多元化合物体系可 以有效调控其禁带宽度。目前, 四元 Bi-M-V-O 体系 的化合物主要有 $\mathrm{BiCdVO}_{5} 、 \mathrm{BiMnVO}_{5} 、 \mathrm{BiM}_{2} \mathrm{VO}_{6}$ $(\mathrm{M}=\mathrm{Zn} 、 \mathrm{Cd}) 、 \mathrm{BiCu}_{2} \mathrm{VO}_{6} 、 \mathrm{BiAe}_{2} \mathrm{VO}_{6}(\mathrm{Ae}=\mathrm{Mg} 、 \mathrm{Ca})$ 等 ${ }^{[18-23]}$ 。其中 $\mathrm{Ae}$ 元素的电正性较强, 会增大体系的 禁带宽度。过渡金属 $\mathrm{Zn}$ 和 $\mathrm{Cd}$ 中满占据的 $\mathrm{nd}$ 轨道 能量较低, ns 空轨道能量较高, 对费米能级附近的 能带结构贡献少，难以调控其禁带宽度。Bhim 等 ${ }^{[24]}$ 采用固相法制备了 $\mathrm{BiCdVO}_{5}$, 其禁带宽度 $(2.45 \mathrm{eV})$ 与 $\mathrm{BiVO}_{4}$ 接近, 且在光催化苯甲醇转化为苯甲醛的 反应中表现出较高的活性和选择性。部分占据的 $\mathrm{Cu}^{2+} 3 \mathrm{~d}$ 轨道和 $\mathrm{Mn}^{2+} 3 \mathrm{~d}$ 轨道可以有效抬升价带能量, 从而降低材料的禁带宽度。Liu 等 ${ }^{[25]}$ 采用固相法制 备了 $\mathrm{BiCu}_{2} \mathrm{VO}_{6}$, 其禁带宽度为 $2.1 \mathrm{eV}$, 且表现出优 于 $\mathrm{BiVO}_{4}$ 的光催化性能。然而, $\mathrm{BiCu}_{2} \mathrm{VO}_{6}$ 的禁带宽 度未在最佳光吸收的范围内 $((1.5 \pm 0.3) \mathrm{eV})$, 光吸收 效率仍较低。图 1 为引入 $\mathrm{Mn}^{2+}$ 导致禁带宽度降低的 示意图。 $\mathrm{MnO}$ 的价带顶为 $\mathrm{Mn} 3 \mathrm{~d}$ 和 $\mathrm{O} 2 \mathrm{p}$ 轨道, 导带 底为 $\mathrm{Mn} 3 \mathrm{~d}$ 轨道, 禁带宽度为 $3.8 \mathrm{eV}^{[26-27]}$; 而 $\mathrm{BiVO}_{4}$ 的价带顶为 $\mathrm{Bi} 6 \mathrm{~s}$ 和 $\mathrm{O} 2 \mathrm{p}$ 轨道, 导带底为 $\mathrm{V} 3 \mathrm{p}$ 轨道, 禁带宽度为 $2.45 \mathrm{eV}^{[28]}$ 。因此, 随着 $\mathrm{Mn}$ 元素的引入,
填充态的 Mn3d 轨道会抬高价带顶能量, 而由 V $3 d$ 组成导带底基本不变。因此, $\mathrm{BiMnVO}_{5}$ 的禁带宽度 小于 $\mathrm{BiVO}_{4}$ 。 $\mathrm{BiMnVO}_{5}$ 为已知化合物, 具有三斜结 构 $(\mathrm{P}-1, a=0.6912 \mathrm{~nm}, b=0.6991 \mathrm{~nm}, c=0.5354 \mathrm{~nm}$, $\alpha=108.55^{\circ}, \beta=95.98^{\circ}, \gamma=109.73^{\circ}$ ), 晶体为橙红色, 可 以吸收更多的可见光, 但其光学性质和光催化性能 尚未见报道。

为此，本工作采用水热法和固相法合成了四 元 $\mathrm{BiMnVO}_{5}$, 并对两种方法合成的样品进行表征 分析, 利用第一性原理计算其电子结构, 并以亚甲 基蓝 (MB) 的降解性能评价分析样品的光催化 性能。

\section{1 实验方法}

\section{1 催化剂的样品制备}

采用水热法合成 $\mathrm{BiMnVO}_{5}$ 催化剂样品。称取 $2 \mathrm{~g}$ 聚乙烯吡咯烷酮(PVP, K30)溶解于 $10 \mathrm{~mL} 1 \mathrm{~mol} / \mathrm{L}$ $\mathrm{HNO}_{3}$ 中形成溶液 $\mathrm{A}$ 。称取 $0.485 \mathrm{~g} \mathrm{Bi}\left(\mathrm{NO}_{3}\right)_{3} \cdot 5 \mathrm{H}_{2} \mathrm{O}$ 和 $0.234 \mathrm{~g} \mathrm{MnCl}_{2} \cdot 6 \mathrm{H}_{2} \mathrm{O}$ 加入 $\mathrm{A}$ 溶液中并摚拌溶解形 成溶液 $\mathrm{B}$ 。称取 $0.117 \mathrm{~g}\left(\mathrm{NH}_{4}\right) \mathrm{VO}_{3}$ 溶解于 $10 \mathrm{~mL}$ 氨 水中形成溶液 $\mathrm{C}$ 。剧烈摚拌下将溶液 $\mathrm{C}$ 缓慢滴加到 溶液 $\mathrm{B}$ 中, 持续搅拌 $30 \mathrm{~min}$, 形成黄色浊液, 用氨 水调节 $\mathrm{pH}=10$ 。将上述浊液转移到 $50 \mathrm{~mL}$ 聚四氟乙 烯的内祄中 (填充率 50\%), 装入水热釜的钢套中并 封紧。该反应在 $200{ }^{\circ} \mathrm{C}$ 下进行, 并保温 $12 \mathrm{~h}$ 。反应 结束后取出反应釜并冷却到室温。离心分离反应后 的浊液, 用去离子水清洗 5 遍。真空干燥后获得 $\mathrm{BiMnVO}_{5}$ 催化剂, 记为 BMVO-H。

为了更好地说明 BMVO-H 催化剂的性质, 分别 采用固相法和水热法合成了 $\mathrm{BiMnVO}_{5}$ 和 $\mathrm{BiVO}_{4}$ 。其 中, 水热法合成 $\mathrm{BiVO}_{4}$ 样品的实验过程与 $\mathrm{BMVO}-\mathrm{H}$ 样品类似, 仅不添加 $\mathrm{MnCl}_{2} \cdot 6 \mathrm{H}_{2} \mathrm{O}$ 。固相法合成 $\mathrm{BiMnVO}_{5}$ 粉末样品的具体合成方法如下：称取 $0.932 \mathrm{~g} \mathrm{Bi}_{2} \mathrm{O}_{3} 、 0.284 \mathrm{~g} \mathrm{MnO} 、 0.364 \mathrm{~g} \mathrm{~V}_{2} \mathrm{O}_{5}$, 并混合

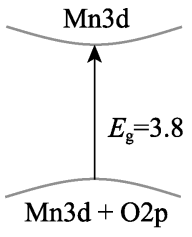

$\mathrm{MnO}$

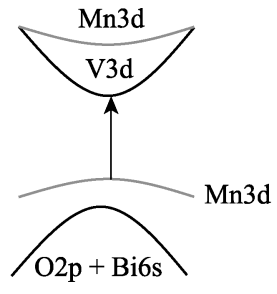

$\mathrm{BiMnVO}_{5}$

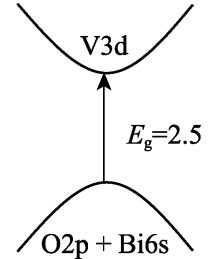

$\mathrm{BiVO}_{4}$
图 $1 \mathrm{MnO} 、 \mathrm{BiVO}_{4}$ 和 $\mathrm{BiMnVO}_{5}$ 能带结构示意图

Fig. 1 Schematic band structure of $\mathrm{MnO}, \mathrm{BiVO}_{4}$ and $\mathrm{BiMnVO}_{5}$ 
均匀。随后将上述混合物转移到一端封口的石英管 中, 在抽真空的情况下 $(0.1 \mathrm{~Pa})$ 封装。随后将封好的 石英管缓慢加热到 $700{ }^{\circ} \mathrm{C}$, 并保温 $1 \mathrm{~d}$ 。自然冷却后, 取出样品, 研磨。重复上述固相反应过程 3 4 次, 获 得 $\mathrm{BiMnVO}_{5}$ 粉末样品, 记为 BMVO-S。

\section{2 催化剂的表征}

在 Phenom ProX 型台式扫描电镜上完成催化剂 样品的扫描电子显微镜(SEM)和 EDX 测试, 灯丝材 料为 $\mathrm{CeB}_{6}$, 加速为 $10 \mathrm{kV}(\mathrm{SEM})$ 和 $15 \mathrm{kV}(\mathrm{EDX})$ 。采 用 Bruker D2 型 X 射线粉末衍射仪测试粉末样品的 $\mathrm{XRD}$ 图谱 $\left(\mathrm{Cu}\right.$ 靶 $\left.\mathrm{K}_{\alpha}, \lambda=0.15406 \mathrm{~nm}\right)$, 采集数据步长 为 $0.02^{\circ}$, 扫描速度为 $6\left(^{\circ}\right) / \mathrm{min}$ 。采用 Kratos Analytical 公司的 Axis Ultra 型光电子能谱仪测试材料 XPS 谱 图, 作为内标的污染碳结合能为 $284.8 \mathrm{eV}$ 。采用 Hitachi U-4100 型紫外可见分光光度计测试粉末样 品的紫外-可见-近红外(UV-Vis-NIR)漫反射光谱图, 测试波长范围在 400 1200 nm 之内。选取全反射的 $\mathrm{BaSO}_{4}$ 粉末作为标准样品。

\section{3 电子结构计算}

采用基于密度泛函理论(DFT)的第一性原理计 算材料的电子结构, 具体的计算操作在 Wien $2 K^{[29]}$ 软 件包中进行。采用 Perdew-Burke-Ernzerhof (PBE)版本 的广义梯度近似(Generalized Gradient Approximation, GGA $)^{[30]}$ 来描述交换相关函数, 噟势选择投影缀加 波(Projector Augmented Wave, PAW) 法 ${ }^{[11-32]}$ 。平面波 的截止能量为 $350 \mathrm{eV}$ 。选取合适的 Monkhorst-Pack 网格对结构进行优化, 结构弛豫的最佳几何位置必 须满足: (1)总能量改变在 $10^{-6} \mathrm{eV} /$ atom之内; (2) 所有 原子位置的 Hellmann-Feynman 力小于 $0.1 \mathrm{eV} / \mathrm{nm}$ 。

\section{4 可见光催化性能测试}

选取亚甲基蓝(MB)的可见光降解反应表征所 合成催化剂的催化性能。为防止催化过程中的热效 应, 利用循环水冷将体系温度固定在室温 $\left(20{ }^{\circ} \mathrm{C}\right)$ 。 $\mathrm{MB}$ 溶液的起始浓度为 $10 \mathrm{mg} / \mathrm{L}$ 。分别称取一定质量 的 $\mathrm{BiVO}_{4} 、$ BMVO-S 和 BMVO-H 催化剂样品, 加入 到 $100 \mathrm{~mL} \mathrm{MB}$ 溶液中(催化剂浓度为 $1 \mathrm{~g} / \mathrm{L}$ ), 并在避 光下摚拌吸附 $3 \mathrm{~h}$ 。待吸附平衡后, 打开氙灯光源进 行光催化反应。采用 $420 \mathrm{~nm}$ 滤光片滤掉紫外光。在 催化过程中, 每隔一定时间取 $5 \mathrm{~mL}$ 悬浊液, 离心分 离出催化剂, 取上层清液测试其吸收光谱。

光催化实验后, 对含有 BMVO-H催化剂样品的 浊液进行离心, 去离子水洗涤至完全去除表面的吸 附物, 真空干燥后, 重复上述光催化性能测试。 BMVO-H 催化剂样品进行 5 次循环测试。

\section{2 结果与讨论}

\section{1 形貌与晶体结构}

相比于固相法的反应温度高、耗时长等缺点, 水 热法可以快速合成高纯度、高结晶度的 $\mathrm{BiMnVO}_{5}$ 。 图 2 为水热法合成 $\mathrm{BiMnVO}_{5}$ 粉体的 SEM 照片和 EDX 元素分布。水热法合成 $\mathrm{BiMnVO}_{5}$ 呈单分散的 花状团簇形貌, 团簇的平均尺寸约为 $20 \mu \mathrm{m}$ 。每个 $\mathrm{BiMnVO}_{5}$ 花状团簇均由柱状晶体聚集而成。EDX 结果表明 $\mathrm{Bi} 、 \mathrm{Mn} 、 \mathrm{~V}$ 和 $\mathrm{O}$ 四种元素均匀分布于花 状团簇中, 元素比例为 $12.6: 11.7: 11.3: 64.4$, 接近 $1: 1: 1: 5$ 。

图 3(a)为水热法和传统固相法合成 $\mathrm{BiMnVO}_{5}$ 粉体的 XRD 图谱, 其中理论衍射峰采用 $\mathrm{BiMnVO}_{5}$ 的晶体结构(ICSD 59720)进行模拟。两种方法合成 $\mathrm{BiMnVO}_{5}$ 粉体的 XRD 图谱均可用理论衍射峰指标 化, 未观测到额外的衍射峰, 证明所得粉末样品为 三斜相 $\mathrm{BiMnVO}_{5}(\mathrm{P}-1, a=0.6912 \mathrm{~nm}, b=0.6991 \mathrm{~nm}$, $\left.c=0.5354 \mathrm{~nm}, \alpha=108.55^{\circ}, \beta=95.98^{\circ}, \gamma=109.73^{\circ}\right)^{[19]}$, 且具有较高的纯度。图 3(b)为三斜相 $\mathrm{BiMnVO}_{5}$ 的晶 体结构。其中, $\mathrm{Mn}$ 原子与六个 $\mathrm{O}$ 原子成键, 形成 $\left[\mathrm{MnO}_{6}\right]^{6-}$ 八面体, 两个 $\left[\mathrm{MnO}_{6}\right]^{6-}$ 八面体通过共边连 接形成 $\left[\mathrm{Mn}_{2} \mathrm{O}_{10}\right]^{16-}$ 二聚体。 $\mathrm{V}$ 原子与四个 $\mathrm{O}$ 原子成键, 形成 $\left[\mathrm{VO}_{4}\right]^{5-}$ 四面体。 $\left[\mathrm{VO}_{4}\right]^{5-}$ 四面体与 $\left[\mathrm{Mn}_{2} \mathrm{O}_{10}\right]^{16-}$ 二 聚体通过氧原子共顶点连接, 形成三维扩展结构。

\section{$2.2 \mathrm{X}$ 射线光电子能谱}

为了探究两种合成方法所得 $\mathrm{BiMnVO}_{5}$ 粉体中 各元素的价态, 对 BMVO-S 和 BMVO-H 样品进行 XPS 表征。图 4(a)为 Bi 的高分辨 XPS 谱图, BMVO-S 和 BMVO-H 样品中 $\mathrm{Bi}$ 离子均出现了位于 159.2 和

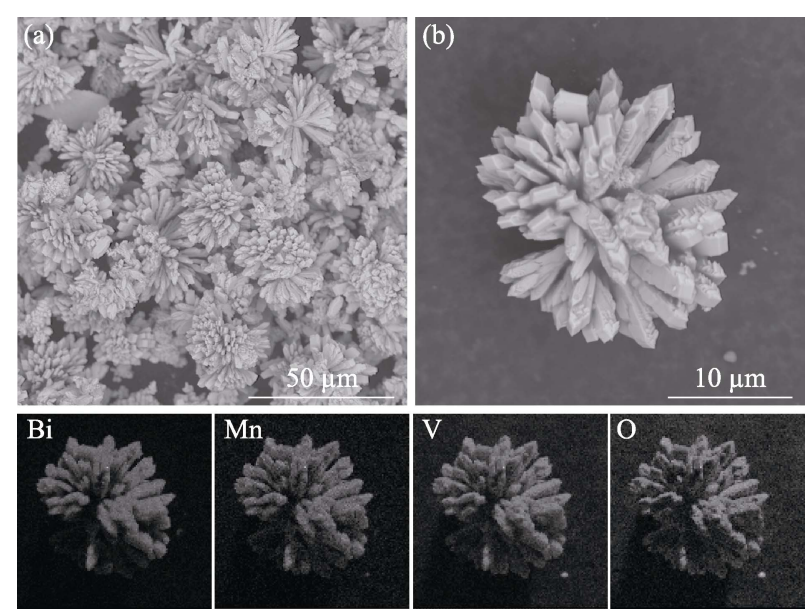

图 2 水热法合成 $\mathrm{BiMnVO}_{5}$ 粉体的 SEM 照片与 EDX 元素 分布

Fig. 2 SEM images and EDX elemental mappings of the hydrothermal synthesized $\mathrm{BiMnVO}_{5}$ powder 

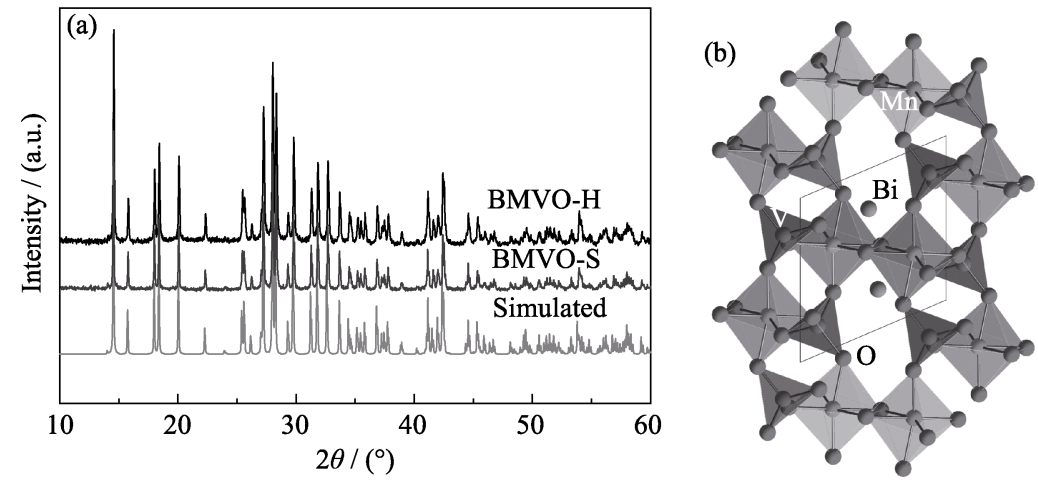

图 3 (a)BiMnVO 5 粉体的 XRD 谱图和(b) $\mathrm{BiMnVO}_{5}$ 的晶体结构

Fig. 3 (a) XRD patterns of the as-synthesized $\mathrm{BiMnVO}_{5}$ powders, and (b) crystal structure of $\mathrm{BiMnVO}_{5}$
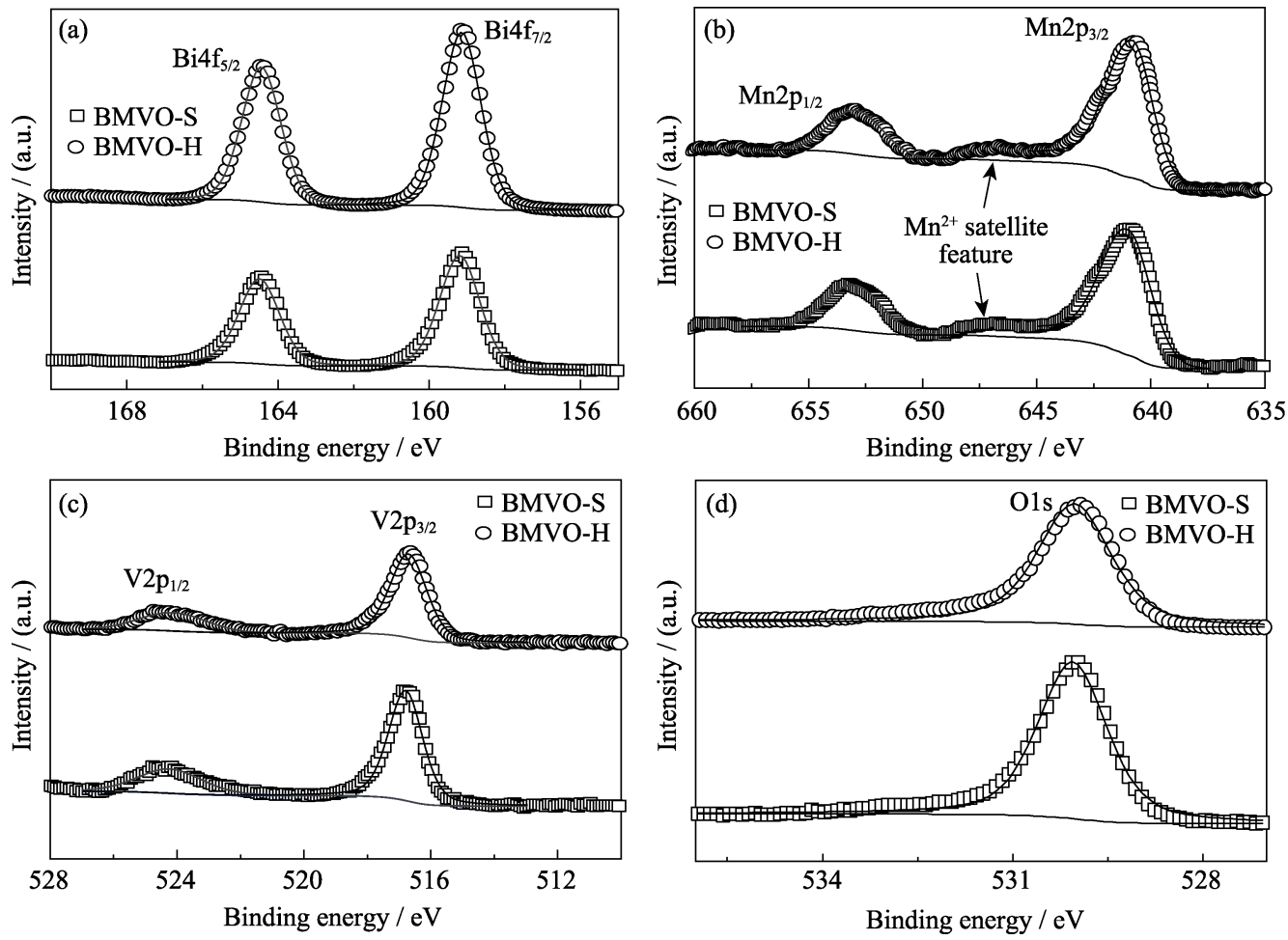

图 $4 \mathrm{BiMnVO}_{5}$ 粉体的(a)Bi4f、(b)Mn2p、(c)V2p 和(d)O1s XPS 图谱

Fig. 4 (a) Bi4f, (b) Mn2p, (c) V2p, and (d) O1s XPS spectra of the as-synthesized BiMnVO 5 powders

$164.5 \mathrm{eV}$ 附近的双峰, 对应于 $\mathrm{Bi}_{4} \mathrm{f}_{7 / 2}$ 和 $\mathrm{Bi}^{4} \mathrm{f}_{5 / 2}$ 的自 旋-轨道分裂峰, 说明两种样品 $\mathrm{Bi}$ 离子均为 +3 价 ${ }^{[33-35]}$ 。 图 4(b)为 Mn 的高分辨 XPS 谱图, 两种样品中 $\mathrm{Mn}$ 离子均出现了位于 640.8 和 $653.1 \mathrm{eV}$ 附近的双峰, 对 应于 $M n 2 p_{3 / 2}$ 和 $M n 2 p_{1 / 2}$ 的分裂峰, 结合能为 $647 \mathrm{eV}$ 左右的卫星峰为 $\mathrm{Mn}^{2+}$ 离子的特征峰, 说明两种样品 中 $\mathrm{Mn}$ 离子均为 +2 价 $^{[36-37]}$ 。图 4(c) 是 $\mathrm{V}$ 的高分辨 XPS 谱图, 图中 V2p 由两个能谱峰组成, 结合能分别位 于 516.7 和 $524.4 \mathrm{eV}$ 左右，对应于 $\mathrm{V} 2 \mathrm{p}_{3 / 2}$ 和 $\mathrm{V} 2 \mathrm{p}_{1 / 2}$ 的分裂峰 ${ }^{[33-35]}$ 。如图 4(d)所示, 两种样品中 $\mathrm{O} 1 \mathrm{~s}$ 能谱 峰均位于 $529.9 \mathrm{eV}$ 左右, 对应于 $\mathrm{BiMnVO}_{5}$ 中的晶格 氧 $\left(\mathrm{O}^{2-}\right)^{[33-35]}$ 。因此, 两种方法合成的 $\mathrm{BiMnVO}_{5}$ 粉体 中 $\mathrm{Bi} 、 \mathrm{Mn} 、 \mathrm{~V} 、 \mathrm{O}$ 的化合价分别为 $+3 、+2 、+5 、-2$ 。

\subsection{UV-Vis-NIR 漫反射谱}

图 5(a)为 $\mathrm{BiVO}_{4} 、 \mathrm{BMVO}-\mathrm{S}$ 和 BMVO-H 的 UVVis-NIR 漫反射光谱图。水热法合成的 $\mathrm{BiVO}_{4}$ 样品 的吸收边位于 $510 \mathrm{~nm}$ ，与文献[2]的报道值相吻合。 相比于 $\mathrm{BiVO}_{4}, \mathrm{BMVO}-\mathrm{S}$ 和 BMVO-H 的吸收边均出 现较大的红移, 位于 $700 \mathrm{~nm}$ 附近。此外, 在 700 $1200 \mathrm{~nm}$ 范围内, BMVO-S 和 BMVO-H 的光吸收能 力明显强于 $\mathrm{BiVO}_{4}$ 。因此, $\mathrm{BiMnVO}_{5}$ 对可见光的利 用率远大于 $\mathrm{BiVO}_{4}$ 。

采用 Kubelka-Munk 方程 ${ }^{[38]}$ 可以将材料的反射 光谱转变为吸收数据, 如式 1 所示。

$$
\mathrm{F}(R)=(1-R)^{n} / 2 R=\alpha / S
$$

其中, $R$ 为反射率; $\alpha$ 为吸收系数; $S$ 为反射系数; $n$ 为 
常数, 对于直接带隙半导体和间接带隙半导体 $n$ 分 别为 0.5 和 2 。本文中 $n$ 取 0.5 (详见电子结构计算), 并以 $[\mathrm{F}(R) h v]$ 对 $h v$ 作图(图 5(b)), 外推切线与横坐 标的交点即为禁带宽度。BMVO-S 和 BMVO-H 的禁 带宽度均为 $1.8 \mathrm{eV}$, 远小于 $\mathrm{BiVO}_{4}(2.45 \mathrm{eV})^{[2] 、}$ $\mathrm{BiCdVO}_{5}(2.45 \mathrm{eV})^{[24]} 、 \mathrm{BiCu}_{2} \mathrm{VO}_{5}(2.1 \mathrm{eV})^{[25]}$ 。

\section{4 电子结构}

为了理解 $\mathrm{BiMnVO}_{5}$ 的可见光吸收特性, 采用第 一性原理计算其电子结构, 如图 6(a)所示。 $\mathrm{BiMnVO}_{5}$ 具有典型的半导体能带结构, 价带顶(VBM)和导带

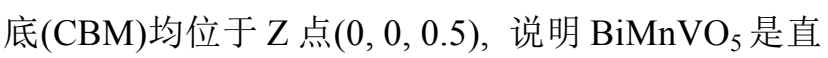
接带隙半导体, 禁带宽度为 $1.28 \mathrm{eV}$, 小于光学带隙, 这是由于 DFT 的计算方法经常低估半导体的禁带 宽度。

仔细分析 $\mathrm{BiMnVO}_{5}$ 的总态密度以及各元素的 态密度分布可以解释引入含锰结构功能区会降低禁 带宽度。如图 6(b) 所示, Mn3d 和 O2p 轨道是价带顶 的主要组成部分, 而 V3d 轨道则是导带底的主要形 成部分。元素 $\mathrm{Bi}$ 的轨道在较大的能量范围内弥散。

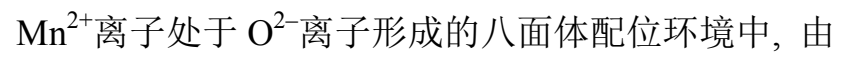
于晶体场䢃裂能的存在, $M n 3 d$ 轨道会分裂成为 $\mathrm{t}_{2 \mathrm{~g}}$ 和 $e_{g}$ 两个部分, 如图 6(b) 所示, 其中 $t_{2 g}$ 位于价带顶, $\mathrm{e}_{\mathrm{g}}$ 位于导带中能量较高的区域。因此, $\mathrm{BiMnVO}_{5}$ 在 光照下的电子跃迁主要发生在 $\mathrm{Mn} 3 \mathrm{~d} / \mathrm{O} 2 \mathrm{p}$ 轨道和 $\mathrm{V} 3 \mathrm{~d}$ 轨道之间。

为了确定 $\mathrm{BiMnVO}_{5}$ 的价带边, 测试了 BMVO-H 样品的 XPS 价带谱, 如图 7 所示。通过外推切线与 横坐标交点的方法确定样品的价带边相对于其费米 能级的位置为 $-1.15 \mathrm{eV}$ 。结合其禁带宽度 $\left(E_{\mathrm{g}}=1.8 \mathrm{eV}\right)$, BMVO-H 样品的价带 (Valence Band, VB)、导带 (Conduction Band, $\mathrm{CB}$ ) 和费米能级 $\left(E_{\mathrm{f}}\right)$ 的相对位置如 图 7 中插图所示。BMVO-H 样品的费米能级接近 $\mathrm{CB}$, 说明其为 $\mathrm{n}$ 型半导体。

\section{5 光催化性能}

由于 $\mathrm{BiMnVO}_{5}$ 可以吸收大部分的可见光, 该样 品可能在可见光降解染料的反应中起到催化剂的作 用。图 8(a)为不同催化剂和催化剂浓度下 MB 溶 液的降解过程。在不添加催化剂的情况下, $4 \mathrm{~h}$ 后, $\mathrm{MB}$ 的降解率仅为 $15 \%$ 。随着 $\mathrm{BiVO}_{4}$ 样品的加入, $\mathrm{MB}$ 的降解速率大幅提升, 催化降解 $4 \mathrm{~h}$ 后, MB 的降解 率为 $80 \%$ 。BMVO-S 和 BMVO-H 催化剂对 MB 的 $4 \mathrm{~h}$ 降解率分别为 $85 \%$ 和 $98 \%$, 优于 $\mathrm{BiVO}_{4}$, 这是由 于 $\mathrm{BiMnVO}_{5}$ 可以吸收更多的可见光。BMVO-H 的 催化活性优于 BMVO-S, 这是因为其花状形貌有助
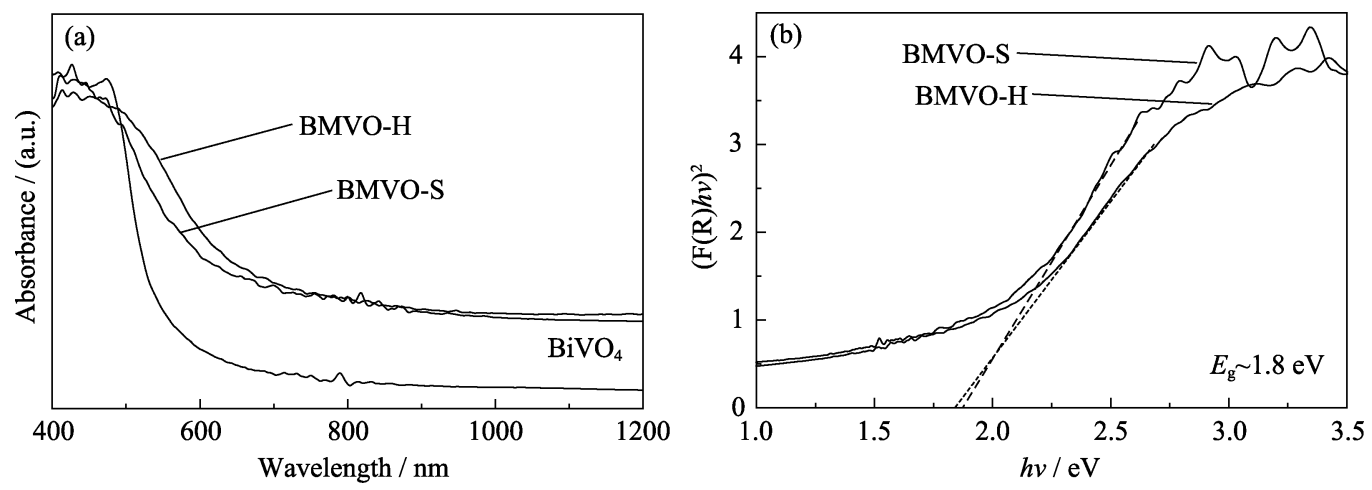

图 $5 \mathrm{BiVO}_{4} 、$ BMVO-S 和 BMVO-H 催化剂的(a)紫外-可见-近红外漫反射光谱和(b) $[\mathrm{F}(R) h v]^{2}$ 随 $h v$ 的变化曲线 Fig. 5 (a) UV-Vis diffuse reflection spectra and (b) $[\mathrm{F}(R) h v]^{2} v s h v$ curves of BMVO-S and BMVO-H photocatalysts
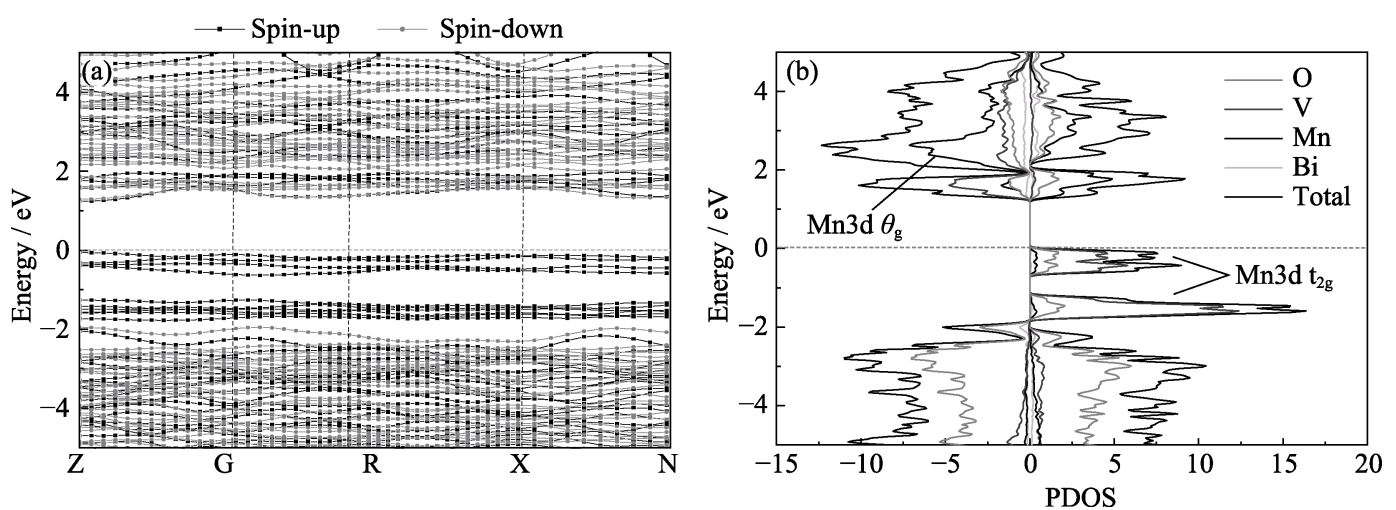

图 $6 \mathrm{BiMnVO}_{5}$ 的能带结构(a)和态密度(b)

Fig. 6 (a) Band structure and (b) density of states of $\mathrm{BiMnVO}_{5}$ 


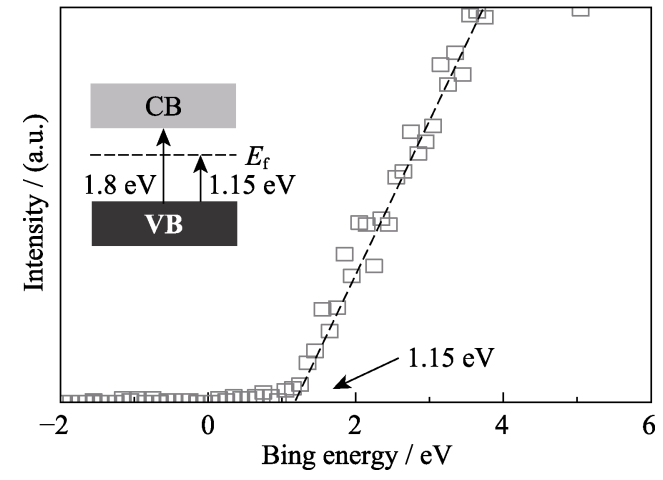

图 7 BMVO-H 样品的 XPS 价带谱(插图为 $\mathrm{VB} 、 \mathrm{CB}$ 和 $E_{\mathrm{f}}$ 的 相对位置)

Fig. 7 XPS valance band of BMVO-H sample with inset showing the relative positions of $\mathrm{VB}, \mathrm{CB}$ and $E_{\mathrm{f}}$

于反应物分子的吸附和扩散，而且便于入射光照射 到更多催化剂表面，从而提高光催化活性。增大 BMVO-H 催化剂浓度可以显著提升 MB 的降解速率, 当催化剂浓度为 $4 \mathrm{~g} / \mathrm{L}$ 时, $2.5 \mathrm{~h}$ 即可实现 $100 \%$ 降解。

为了研究催化反应过程中的活性物, 以 BMVO-H 样品的催化活性作为参考, 分别向催化反应体系中 引入异丙醇(IPA)、草酸铵 $(\mathrm{AO})$ 和对苯醌(BQ)作为羟 基自由基 $(\cdot \mathrm{OH})$ 、空穴 $\left(\mathrm{h}^{+}\right)$和超氧自由基 $\left(\cdot \mathrm{O}_{2}^{-}\right)$的捕捉

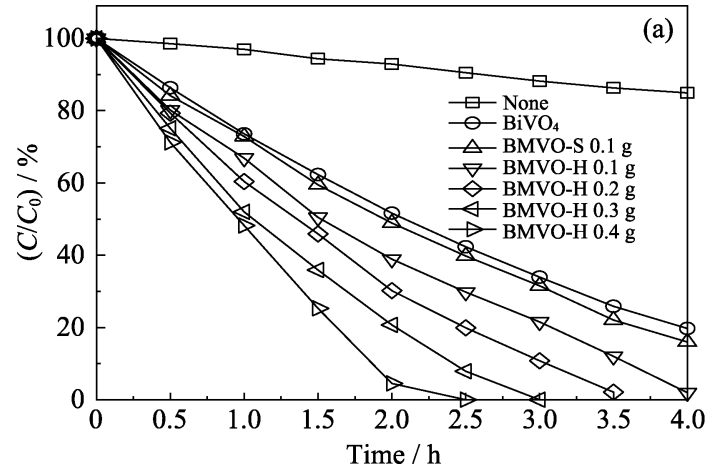

剂。如图 8(b)所示, 加入苯醌后, BMVO-H 催化活性 变化不大，催化反应 $4 \mathrm{~h}$ 后，亚甲基蓝降解率为 $88 \%$ 。而加入异丙醇或草酸铵后, 样品催化活性大 幅降低，亚甲基蓝的相应降解率分别为 57\%和 24\%, 证明羟基自由基和光生空穴是该光催化反应的主要 活性物。

为了评估 $\mathrm{BiMnVO}_{5}$ 光催化剂的稳定性, 对最优 催化性能的 BMVO-H 样品进行了 5 次可见光催化降 解 MB 的重复试验, 如图 9 所示。结果表明, 5 次重 复催化反应后 MB 的降解率分别为 $98 \%$ 、96\%、93\%、 $88 \%$ 和 $85 \%$ 。重复试验后, BMVO-H 样品维持原有的 花状形貌不变(图 9(b)), 且催化剂的结构基本保持不 变(图 9(c))。 $\mathrm{BiMnVO}_{5}$ 表现出优越的稳定性, 具有实 际应用前景。

\section{3 结论}

采用水热法和高温固相法成功合成了四元 $\mathrm{BiMnVO}_{5}$ 可见光催化剂。研究结果表明, 水热法可 以快速合成高纯度、高结晶度的 $\mathrm{BiMnVO}_{5}$, 样品呈 现单分散的花状团簇的形貌。紫外-可见漫反射谱分

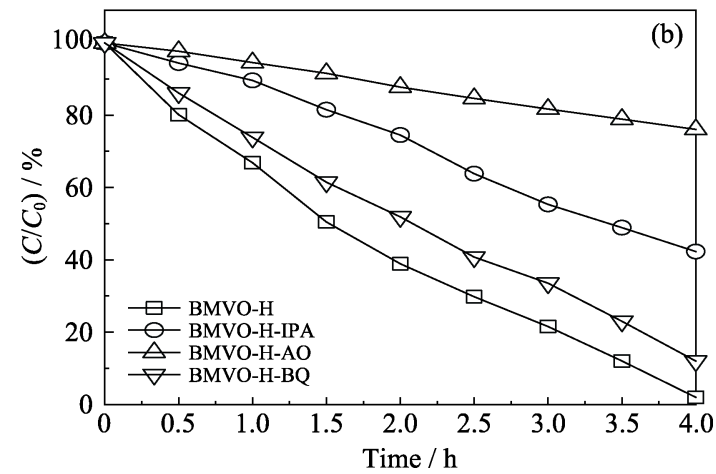

图 8 (a)不同催化条件下 MB 浓度随时间变化曲线和(b)加入不同捕捉剂后 MB 浓度随时间变化曲线

Fig. 8 (a) Curves of MB concentration $v s$ time under different catalytic conditions, and (b) curves of MB concentration $v s$ time with different scavengers
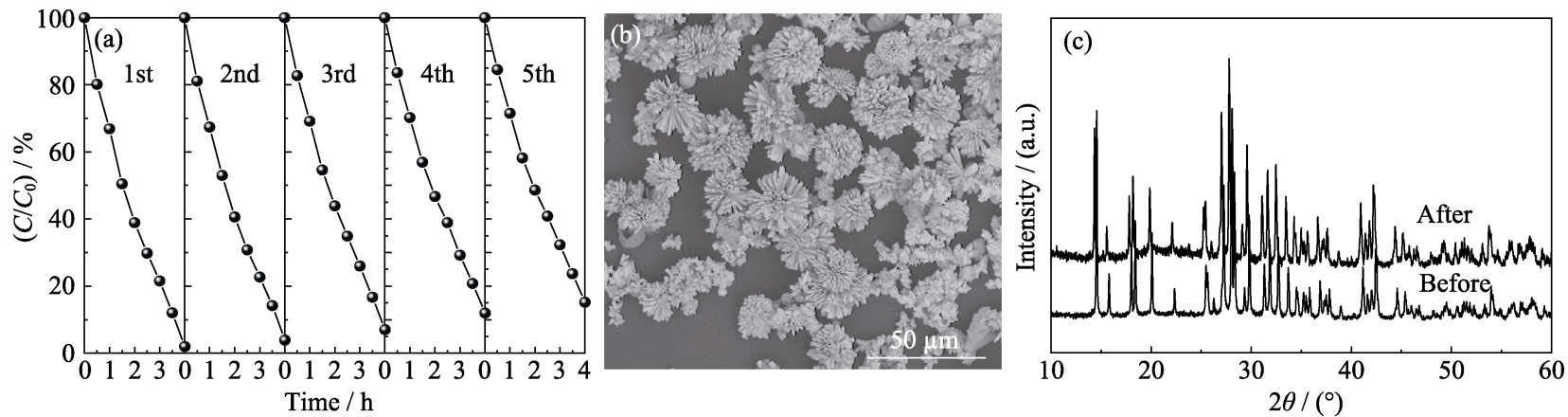

图 9 (a)BMVO-H 样品可见光催化降解 MB 的重复试验, (b)5 次循环后 BMVO-H 样品的 SEM 照片和 (c)5 次循环前后 BMVO-H 样品的 XRD 图谱

Fig. 9 (a) Repeated test for visible light degradation of MB for BMVO-H sample, (b) SEM image of BMVO-H sample after 5 cycles, and (c) XRD patterns of BMVO-H sample before and after 5 cycles 
析发现 $\mathrm{BiMnVO}_{5}$ 可以吸收大部分可见光, 禁带宽度 为 $1.8 \mathrm{eV}$ ，远小于 $\mathrm{BiVO}_{4}(2.45 \mathrm{eV})$ 。第一性原理计算 结果表明, $\mathrm{BiMnVO}_{5}$ 为典型的直接带隙半导体, 光照 下电子跃迁发生在 $\mathrm{Mn} 3 \mathrm{~d} / \mathrm{O} 2 \mathrm{p}$ 和 $\mathrm{V} 3 \mathrm{~d}$ 轨道之间, 引入 含 $\mathrm{Mn}$ 堆积基元造成价带能量升高是其禁带宽度小 于 $\mathrm{BiVO}_{4}$ 的根本原因。光催化实验结果显示, 水热 法合成的 $\mathrm{BiMnVO}_{5}$ 催化剂的催化活性最高, 可见光 照射 $4 \mathrm{~h}$ 后亚甲基蓝的降解率为 $98 \%$, 比 $\mathrm{BiVO}_{4}$ 提升 了 $18 \%$ 。光催化机理研究表明羟基自由基和光生空 穴是催化反应过程中的主要活性物质。循环实验表 明催化剂具有优异的稳定性。本研究为进一步开发 基于 $\mathrm{BiVO}_{4}$ 的高效、高稳定的窄带隙可见光催化剂 提供了独特的解决思路。

\section{参考文献:}

[1] ZHAO C, CHEN Z, SHI R, et al. Recent advances in conjugated polymers for visible-light-driven water splitting. Advanced Materials, 2020, 32(28): 1907296

[2] KIM T W, CHOI K S. Nanoporous $\mathrm{BiVO}_{4}$ photoanodes with duallayer oxygen evolution catalysts for solar water splitting. Science, 2014, 343(6174): 990-994.

[3] YUAN D, SUN M, TANG S, et al. All-solid-state $\mathrm{BiVO}_{4} / \mathrm{ZnIn}_{2} \mathrm{~S}_{4}$ $Z$-scheme composite with efficient charge separations for improved visible light photocatalytic organics degradation. Chinese Chemical Letters, 2020, 31(2): 547-550.

[4] CHEN Q, CHENG X, LONG H, et al. A short review on recent progress of $\mathrm{Bi} /$ semiconductor photocatalysts: the role of $\mathrm{Bi}$ metal. Chinese Chemical Letters, 2020, 31(10): 2583-2590.

[5] TOKUNAGA S, KATO H, KUDO A. Selective preparation of monoclinic and tetragonal $\mathrm{BiVO}_{4}$ with scheelite structure and their photocatalytic properties. Chemistry of Materials, 2001, 13(12): 4624-4628.

[6] ZHOU B, ZHAO X, LIU H, et al. Visible-light sensitive cobaltdoped $\mathrm{BiVO}_{4}\left(\mathrm{Co}-\mathrm{BiVO}_{4}\right)$ photocatalytic composites for the degradation of methylene blue dye in dilute aqueous solutions. Applied Catalysis B: Environmental, 2010, 99(1): 214-221.

[7] HE B, LI Z, ZHAO D, et al. Fabrication of porous Cu-doped $\mathrm{BiVO}_{4}$ nanotubes as efficient oxygen-evolving photocatalysts. ACS Applied Nano Materials, 2018, 1(6): 2589-2599.

[8] REGMI C, KSHETRI Y. K, KIM T H, et al. Visible-light-induced $\mathrm{Fe}$-doped $\mathrm{BiVO}_{4}$ photocatalyst for contaminated water treatment. Molecular Catalysis, 2017, 432: 220-231.

[9] REGMI C, KSHETRI Y K, KIM T H, et al. Fabrication of Ni-doped $\mathrm{BiVO}_{4}$ semiconductors with enhanced visible-light photocatalytic performances for wastewater treatment. Applied Surface Science, 2017, 413: 253-265.

[10] LUO W, LI Z, YU T, et al. Effects of surface electrochemical pretreatment on the photoelectrochemical performance of Modoped $\mathrm{BiVO}_{4}$. The Journal of Physical Chemistry C, 2012, 116(8): 5076-5081.

[11] LUO W, YANG Z, LI Z, et al. Solar hydrogen generation from seawater with a modified $\mathrm{BiVO}_{4}$ photoanode. Energy \& Environmental Science, 2011, 4(10): 4046-4051.

[12] ZHONG D K, CHOI S, GAMELIN D R. Near-complete suppression of surface recombination in solar photoelectrolysis by "Co-Pi" catalyst-modified W:BiVO ${ }_{4}$. Journal of the American Chemical Society, 2011, 133(45): 18370-18377.

[13] ZHONG X, HE H, YANG M, et al. $\mathrm{In}^{3+}$-doped $\mathrm{BiVO}_{4}$ photoanodes with passivated surface states for photoelectrochemical water oxidation. Journal of Materials Chemistry A, 2018, 6(22): 10456-10465.

[14] USAI S, OBREGÓN S, BECERRO A I, et al. Monoclinic-tetragonal heterostructured $\mathrm{BiVO}_{4}$ by yttrium doping with improved photocatalytic activity. The Journal of Physical Chemistry C, 2013, 117(46): 24479-24484.
[15] GOVINDARAJU G V, MORBEC J M, GALLI G A, et al. Experimental and computational investigation of lanthanide ion doping on $\mathrm{BiVO}_{4}$ photoanodes for solar water splitting. The Journal of Physical Chemistry C, 2018, 122(34): 19416-19424.

[16] LUO Y, TAN G, DONG G, et al. Structural transformation of $\mathrm{Sm}^{3+}$ doped $\mathrm{BiVO}_{4}$ with high photocatalytic activity under simulated sun-light. Applied Surface Science, 2015, 324: 505-511.

[17] BAEK J H, GILL T M, ABROSHAN H, et al. Selective and efficient Gd-Doped $\mathrm{BiVO}_{4}$ photoanode for two-electron water oxidation to $\mathrm{H}_{2} \mathrm{O}_{2}$. ACS Energy Letters, 2019, 4(3): 720-728.

[18] RADOSAVLJEVIC I, HOWARD J A K, SLEIGHT A W. Synthesis and structure of two new bismuth cadmium vanadates, $\mathrm{BiCdVO}_{5}$ and $\mathrm{BiCd}_{2} \mathrm{VO}_{6}$, and structures of $\mathrm{BiCa}_{2} \mathrm{AsO}_{6}$ and $\mathrm{BiMg}_{2} \mathrm{PO}_{6}$. International Journal of Inorganic Materials, 2000, 2(6): 543-550.

[19] XUN X, YOKOCHI A, SLEIGHT A W. Synthesis and structure of $\mathrm{BiMnVO}_{5}$ and $\mathrm{BiMnAsO}_{5}$. Journal of Solid State Chemistry, 2002, 168(1): $224-228$

[20] ELIZIARIO NUNES S, WANG C H, SO K, et al. Bismuth zinc vanadate, $\mathrm{BiZn}_{2} \mathrm{VO}_{6}$ : new crystal structure type and electronic structure. Journal of Solid State Chemistry, 2015, 222: 12-17.

[21] RADOSAVLJEVIC I, EVANS J S O, SLEIGHT A W. Synthesis and structure of bismuth copper vanadate, $\mathrm{BiCu}_{2} \mathrm{VO}_{6}$. Journal of Solid State Chemistry, 1998, 141(1): 149-154.

[22] HUANG J, SLEIGHT A W. Synthesis, crystal structure, and optical properties of a new bismuth magnesium vanadate: $\mathrm{BiMg}_{2} \mathrm{VO}_{6}$. Journal of Solid State Chemistry, 1992, 100(1): 170-178.

[23] RADOSAVLJEVIC I, EVANS J S O, SLEIGHT A W. Synthesis and structure of $\mathrm{BiCa}_{2} \mathrm{VO}_{6}$. Journal of Solid State Chemistry, 1998, 137(1): 143-147.

[24] BHIM A, SASMAL S, GOPALAKRISHNAN J, et al. Visiblelight-activated $\mathrm{C}-\mathrm{C}$ bond cleavage and aerobic oxidation of benzyl alcohols employing $\mathrm{BiMXO}_{5}(\mathrm{M}=\mathrm{Mg}, \mathrm{Cd}, \mathrm{Ni}, \mathrm{Co}, \mathrm{Pb}, \mathrm{Ca}$ and $\mathrm{X}=\mathrm{V}$, P). Chemistry - An Asian Journal, 2020, 15(19): 3104-3115.

[25] LIU H, NAKAMURA R, NAKATO Y. Bismuth-copper vanadate $\mathrm{BiCu}_{2} \mathrm{VO}_{6}$ as a novel photocatalyst for efficient visible-light-driven oxygen evolution. ChemPhysChem, 2005, 6(12): 2499-2502.

[26] VAN ELP J, POTZE R H, ESKES H, et al. Electronic structure of MnO. Physical Review B, 1991, 44(4): 1530-1537.

[27] MASSIDDA S, CONTINENZA A, POSTERNAK M, et al. Bandstructure picture for $\mathrm{MnO}$ reexplored: a model $G W$ calculation. Physical Review Letters, 1995, 74(12): 2323-2326.

[28] COOPER J K, GUL S, TOMA F M, et al. Electronic structure of monoclinic $\mathrm{BiVO}_{4}$. Chemistry of Materials, 2014, 26(18): $5365-5373$

[29] BLAHA P, SCHWARZ K, MADSEN G K H, et al. WIEN2k, an augmented plane wave+ local orbitals program for calculating crystal properties. 2001.

[30] PERDEW J P, BURKE K, ERNZERHOF M. Generalized gradient approximation made simple. Physical Review Letters, 1996, 77(18): 3865-3868.

[31] BLÖCHL P E. Projector augmented-wave method. Physical Review B, 1994, 50(24): 17953-17979.

[32] KRESSE G, JOUBERT D. From ultrasoft pseudopotentials to the projector augmented-wave method. Physical Review B, 1999, 59(3): 1758-1775.

[33] JIANG Z, LIU Y, JING T, et al. Enhancing the photocatalytic activity of $\mathrm{BiVO}_{4}$ for oxygen evolution by Ce doping: $\mathrm{Ce}^{3+}$ ions as hole traps. The Journal of Physical Chemistry C, 2016, 120(4): 2058-2063.

[34] PALANISELVAM T, SHI L, METTELA G, et al. Vastly enhanced $\mathrm{BiVO}_{4}$ photocatalytic OER performance by $\mathrm{NiCoO}_{2}$ as cocatalyst. Advanced Materials Interfaces, 2017, 4(19): 1700540.

[35] YAO X, ZHAO X, HU J, et al. The self-passivation mechanism in degradation of $\mathrm{BiVO}_{4}$ photoanode. iScience, 2019, 19: 976-985.

[36] BIESINGER M C, PAYNE B P, GROSVENOR A P, et al. Resolving surface chemical states in XPS analysis of first row transition metals, oxides and hydroxides: $\mathrm{Cr}, \mathrm{Mn}, \mathrm{Fe}, \mathrm{Co}$ and Ni. Applied Surface Science, 2011, 257(7): 2717-2730.

[37] LI M, LEI W, YU Y, et al. High-performance asymmetric supercapacitors based on monodisperse $\mathrm{MnO}$ nanocrystals with high energy densities. Nanoscale, 2018, 10(34): 15926-15931.

[38] KORTÜM G, BRAUN W, HERZOG G. Principles and techniques of diffuse-reflectance spectroscopy. Angewandte Chemie International Edition, 1963, 2(7): 333-341. 